\title{
A simple method to simulate shrinkage-induced cracking in cement-based composites by lattice-type modeling
}

\author{
J. X. Liu - Z. Y. Zhao - S. C. Deng · N. G. Liang
}

Received: 18 June 2008 / Accepted: 20 July 2008 / Published online: 15 August 2008

(C) Springer-Verlag 2008

\begin{abstract}
A new numerical procedure is proposed to investigate cracking behaviors induced by mismatch between the matrix phase and aggregates due to matrix shrinkage in cement-based composites. This kind of failure processes is simplified in this investigation as a purely spontaneous mechanical problem, therefore, one main difficulty during simulating the phenomenon lies that no explicit external load serves as the drive to propel development of this physical process. As a result, it is different from classical mechanical problems and seems hard to be solved by using directly the classical finite element method (FEM), a typical kind of "load $\rightarrow$ medium $\rightarrow$ response" procedures. As a solution, the actual mismatch deformation field is decomposed into two virtual fields, both of which can be obtained by the classical FEM. Then the actual response is obtained by adding together the two virtual displacement fields based on the principle of superposition. Then, critical elements are detected successively by the event-by-event technique. The micro-structure of composites is implemented by employing the generalized beam (GB) lattice model. Numerical examples are given to show the effectiveness of the method, and detailed discussions are conducted on influences of material properties.
\end{abstract}

J. X. Liu $(\varangle) \cdot$ Z. Y. Zhao

School of Civil and Environmental Engineering,

Nanyang Technological University, Singapore 639798, Singapore

e-mail: taibaijinxing@gmail.com

\section{S. C. Deng}

Key Laboratory of High Temperature Gas Dynamics (LHD), Institute of Mechanics, Chinese Academy of Sciences, 100080 Beijing, China

\section{N. G. Liang}

State Key Laboratory of Nonlinear Mechanics (LNM), Institute of Mechanics, Chinese Academy of Sciences, 100080 Beijing, China
Keywords Shrinkage-induced cracking .

Mismatch deformation - Generalized beam lattice model .

Cement-based composites $\cdot$ Mohr-Coulumb failure criterion

\section{Introduction}

Fracture mechanics has mainly focused on materials' failures under external mechanical loads. Failures, however, sometimes happen without explicit mechanical loads. For example, many shrinkage-induced micro- or even macrolevel cracks can be found in an unloaded and unstrained concrete. Because shrinkage is not completely evitable, most concrete specimens for experimental use in laboratories are actually systems filled with pre-existing shrinkage-induced cracks (see for instance [12,15,26,29]). Although these cracks are also micro-level entities as well as aggregates, influences of shrinkage-induced cracks on macro-properties have been rarely considered directly in the existing micro-mechanical models. Usually, this kind of influences is represented by some "black-box" material properties, such as softening curves of matrix and interface zones [13]. Nevertheless, an alternative opinion also seems interesting: to investigate the growth processes of shrinkage-induced cracks, especially when considering the difficulties of detecting these micro-cracks in real physics experiments until now [15].

Although there is a need for understanding the formation and propagation of these pre-existing cracks, advances in this area have been limited greatly by the complex of the problem. On one hand, the complexity lies that factors causing shrinkage deformation include temperature, moisture transport and so on, leading to a multi-physics problem even coupling chemical reactions [8]. On the other hand, shrinkage processes are nearly stochastic due to irregular characteristics of environmental changes and mankind activities. 
Furthermore, the time scale for drying of cement-based composites is at least $\sim$ month [3], leading to a substantial increase in computational effort. As a result, a direct simulation becomes extremely time-consuming and complex. Bolander and Berton [3] coupled the random lattice modeling of moisture movement with the rigid-body-spring networks (RBSN), and took it as a time-dependent process, therefore, a substantial increase in computational effort occurs.

However, the problem may get simple and solvable from a different viewpoint. From the above analyses, the shrinkageinducing factors complicate the investigated problem very much. Nevertheless, cracks are related directly to mismatch deformation due to shrinkage. Therefore, when our aim is to analyze the crack patterns instead of thermal and moist processes, it seems reasonable that shrinkage-induced cracking can be simulated by introducing directly matrix shrinkage into the paste-aggregate system, without account of the shrinkage-inducing factors.

Mismatch deformation seems not to be a classical mechanical problem in some sense. In classical mechanical analyses, medium's response can be solved if external loads are applied. When mismatch deformation happens, however, it is hard to say how much the external load is. Therefore, the simplified shrinkage-induced cracking can be regarded as a spontaneous process without external mechanical loads. This feather makes difficult the direct use of the finite element method (FEM), based on the idea of classical mechanical analysis, i.e. "load $\rightarrow$ medium $\rightarrow$ response" procedure.

In this paper, a simple method is proposed to solve the mismatch deformation problem. The basic idea is to decompose the problem into two classical mechanical ones, which can be easily solved by the classical FEM procedure. In each of these two elementary problems, no failure is permitted even though failure will happen in equivalent real physics experiments. In this sense, both of the elementary problems are virtual.

Here, the micro-structure model of concrete is described by the GB lattice model, a kind of lattice-type model proposed recently by Liu et al. [18-20]. Lattice-type models have a history of several decades. These models are based on the atomic lattice models of materials in principle [22,23]. This kind of models has been successfully used for solving classical problems of elasticity [10]. More recently, it has been employed for simulating the progressive failure in heterogeneous brittle media, especially by theoretical physicists $[1,9]$. To investigate fracture processes in concrete, many different types of lattice models have been developed in the past. As has been generally accepted, it is necessary to project the material structure directly on to the lattice in order to obtain more realistic results [13,30]. Schorn and Rode [28] and Bazant et al. [1] used truss elements, which require some numerical measures to exclude numerical instability during fracture propagation. The virtual internal bond (VIB) model proposed by Gao and Klein [7] also seems similar to the truss-type lattice from the viewpoint of numerical simulation. Schlangen and van Mier [27] adopted Euler-Bernoulli beams of Hughes et al. [11]. Bolander and Saito [2] developed a kind of spring element, which is equivalent to the Euler-Bernoulli beam element in a special parameter setting. Generally, the Timoshenko beam is more proper than the Euler-Bernoulli beam as each beam in the lattice is short and deep [13]. Truss network corresponds to the classical continuum, while beam lattice corresponds to the micro-polar continuum.

On the other hand, lattice models work best if the material may be represented naturally by a system of discrete units interacting through truss, beams or other kinds of twonode elements. Spatial trusses and frameworks have been the primary material systems thus modeled. Currently, there is mounting interest in the potential offered by materials with internal lattice-construction for use in high-performance load-bearing applications. A typical aim is to develop lightweight structures that are adequately stiff and strong. Deshpande et al. [5] indicated the attracting effective properties of the octet-truss lattice material. McKown et al. [21] conducted compression experiments on lattice structures by applying quasi-static and blast loading.

Fracture processes in heterogeneous materials such as rock and concrete are very difficult to interpret without appealing to their micro-structures. The beam lattice model allows for a straightforward implementation of the heterogeneity of the three-phase system: aggregate, matrix and interface between them $[13,20]$. The interfacial phase is the weakest among the three phases. One important reason for the degradation in strength of interfacial phase is that shrinkage causes many micro-level failures around aggregates [4]. In this paper, the aim is to study the shrinkage-induced cracking, in other words the producing process of the weakest interfacial phase. Therefore, it is reasonable to assign a bigger strength value to the matrix surrounding aggregates, which was taken as interface zones in former lattice type modeling [30], and is also regarded as matrix in this study without special announcement.

The two-dimensional GB lattice model is employed in this paper. Of course, the 3D model is a more realistic approximation of materials because the micro-structure in concrete is $3 \mathrm{D}$ in nature. However, the calibration of elemental parameters remains a crucial difficulty even though the difficulty in computational effort can be overcome with the rapid development of computer hardware. In fact, we are investigating the 3D modeling which will be a future topic. Furthermore, the main aim in this study is to propose a new method to simulate the simplified shrinkage cracking process, whose basic idea is independent of the chosen model.

To solve the problem of computational effort, the generalized beam (GB) lattice model was developed by Liu et al. [18]. The key idea of GB lattice is the proposal of a kind of 
two-node and three-segment element. In this paper, in order to obtain more detailed crack growth, numerical simulations are all conducted based on the GB lattice model proposed by Liu et al. [20] where every big aggregate is simulated by dozens of nodes, which is very similar to the classical beam lattices.

The paper is structured as follows. The basic theory of the GB lattice model is recalled in Sect. 2. The new method to simulate mismatch deformation is introduced in Sect. 3. In Sect. 4, the global numerical procedure is given, then MohrCoulumn criterion and event-by-event algorithm are recalled. In Sect. 5, several numerical examples are given to verify the effectiveness of the procedure and to analyze influences of different factors on the shrinkage-induced cracking. The paper ends with conclusions in Sect. 6.

\section{GB lattice model}

In this model proposed by Liu et al. [18-20], a kind of twonode and three-phase elements has been developed. Every element is composed of three beams, which can be aggregatephase, matrix-phase or interface-phase independently (Fig. 1). As a result, an aggregate can be simulated in principle by a single node in the GB lattice, instead of 10-100 nodes in other beam lattices. Therefore, the computational effort is reduced greatly.

In the three-fragment GB element, every fragment is regarded as a beam. These beams can be taken as Euler-Bernoulli beams, Timoshenko beams or beams proposed by Bolander and Saito [2]. No matter which beam theory is adopted, the beam stiffness matrices have the following common expression (Fig. 2):

$\left\{\begin{array}{l}Q_{1} \\ N_{1} \\ M_{1} \\ Q_{2} \\ N_{2} \\ M_{2}\end{array}\right\}=\underbrace{\left[\begin{array}{llllll}\mathrm{M}_{11} & 0 & -\mathrm{M}_{34} & -\mathrm{M}_{11} & 0 & -\mathrm{M}_{34} \\ & \mathrm{M}_{22} & 0 & 0 & -\mathrm{M}_{22} & 0 \\ & & \mathrm{M}_{33} & \mathrm{M}_{34} & 0 & \mathrm{M}_{36} \\ & & & \mathrm{M}_{11} & 0 & \mathrm{M}_{34} \\ & & \mathrm{SYM} & & \mathrm{M}_{22} & 0 \\ & & & & & \mathrm{M}_{33}\end{array}\right]}_{M}$

$$
\times\left\{\begin{array}{l}
u_{1} \\
v_{1} \\
\varphi_{1} \\
u_{2} \\
v_{2} \\
\varphi_{2}
\end{array}\right\}
$$

where, $\mathbf{F}_{12}=\left\{Q_{1} N_{1} M_{1} Q_{2} N_{2} M_{2}\right\}^{\mathrm{T}}$ and $\mathbf{u}_{12}=$ $\left\{u_{1} v_{1} \varphi_{1} u_{2} v_{2} \varphi_{2}\right\}^{\mathrm{T}}$ are the generalized force vector and the generalized displacement vector, respectively. (a)

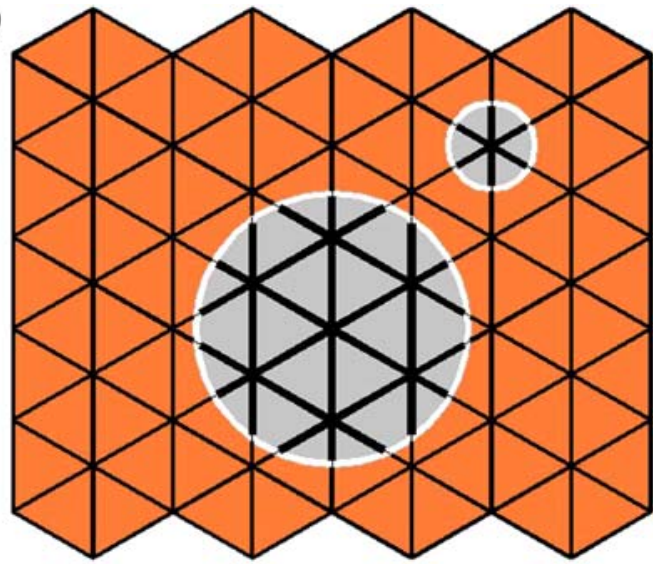

(b)

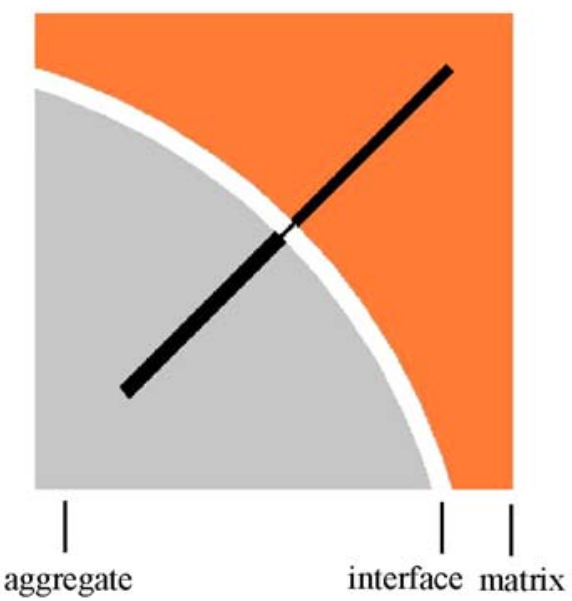

Fig. 1 A GB lattice: a projection of particle structure onto the lattice, b sketch-map of composition of an aggregate-interface-matrix element

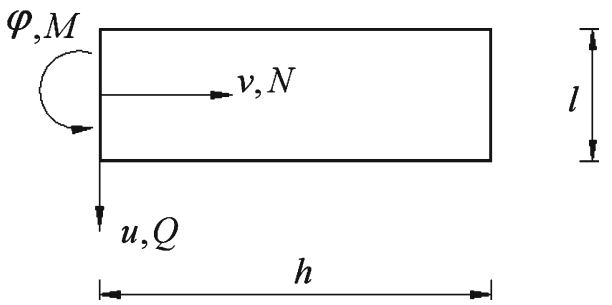

Fig. 2 Kinematics and statics of a beam

For the Euler-Bernoulli beam [13]:

$$
\begin{aligned}
& \mathrm{M}_{11}=\frac{12 E^{(b)} I}{h^{3}}, \quad \mathrm{M}_{34}=\frac{6 E^{(b)} I}{h^{2}}, \quad \mathrm{M}_{22}=\frac{E^{(b)} A}{h}, \\
& \mathrm{M}_{33}=\frac{4 E^{(b)} I}{h}, \quad \mathrm{M}_{36}=\frac{2 E^{(b)} I}{h}
\end{aligned}
$$

For the Timoshenko beam [31]:

$$
\begin{aligned}
& \mathrm{M}_{11}=\frac{12 E^{(b)} I}{(1+b) h^{3}}, \quad \mathrm{M}_{34}=\frac{6 E^{(b)} I}{(1+b) h^{2}}, \quad \mathrm{M}_{22}=\frac{E^{(b)} A}{h}, \\
& \mathrm{M}_{33}=\frac{E^{(b)} I(4+b)}{h(1+b)}, \quad \mathrm{M}_{36}=\frac{E^{(b)} I(2-b)}{h(1+b)}
\end{aligned}
$$


where, $E^{(b)}$ is the Young's Modulus; $t^{(b)}, h$ and $l$ are, respectively, the thickness, the span and the height of the Timoshenko beam; $A=t^{(b)} l$ is the cross-section area; $I=$ $t^{(b)} l^{3} / 12$ is the moment of inertia; $b=a E^{(b)} l^{2} / G^{(b)} h^{2}$ is the dimensionless parameter in Timoshenko beam theory; $G^{(b)}=E^{(b)} / 2\left(1+v^{(b)}\right)$ is the modulus of rigidity, where $v^{(b)}$ is the Poisson's ratio.

For the beam studied in Bolander and Saito [2]:

$\mathrm{M}_{11}=\frac{G^{(b)} A}{h}, \quad \mathrm{M}_{34}=\frac{G^{(b)} A}{2}, \quad \mathrm{M}_{22}=\frac{E^{(b)^{\prime}} A}{h}$,

$\mathrm{M}_{33}=\frac{G^{(b)} A h}{4}+\frac{E^{(b) \prime} I}{h}, \quad \mathrm{M}_{36}=\frac{G^{(b)} A h}{4}-\frac{E^{(b) \prime} I}{h}$

where, $E^{(b) \prime}=E^{(b)} /\left[1-\left(v^{(b)}\right)^{2}\right]$.

The relationship between the lattice and its continuum equivalent is obtained based on the equivalence of strain energy stored in a unit cell of a lattice with its continuum counterpart. The calibration results for a triangular GB lattice are listed as follows.

For the Euler-Bernoulli beam:

$E^{(b)}=\frac{E}{\sqrt{3}}\left(\frac{l}{h}\right)^{-1} \frac{t}{t^{(b)}} \frac{12+\left(\frac{l}{h}\right)^{2}}{4+\left(\frac{l}{h}\right)^{2}}, \quad v=\frac{4-\left(\frac{l}{h}\right)^{2}}{12+\left(\frac{l}{h}\right)^{2}}$.

Note that the Poisson's ratio for Euler-Bernoulli beams is always zero.

For the Timoshenko beam:

$E^{(b)}=\frac{2}{\sqrt{3}}\left(\frac{l}{h}\right)^{-1} \frac{t}{t^{(b)}} \frac{E}{1-v}$,

$v^{(b)}=\frac{5(1+v)}{11(1-3 v)}-\frac{20}{11}\left(\frac{l}{h}\right)^{-2}-\frac{12}{11}$.

For the beam studied in Bolander and Saito [2]:

$E^{(b)}=E, \quad v^{(b)}=v$

The stiffness matrix of a GB element is expressed as functions of material and geometry parameters of its three beams. Take the element shown in Fig. 3 as an example, let $\mathbf{R}$ be the matrix relating the displacement vector $\mathbf{u}_{i j}=$

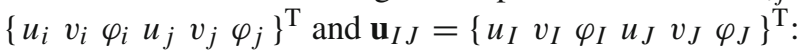

$\mathbf{u}_{I J}=\mathbf{R} \mathbf{u}_{i j}$.

Then the stiffness matrix of the GB element $\mathbf{K}$ can be expressed in the form

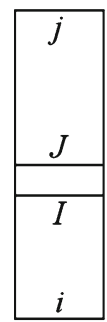

(a)

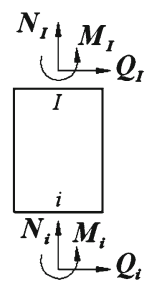

(b)

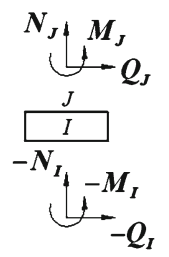

(c) (d)

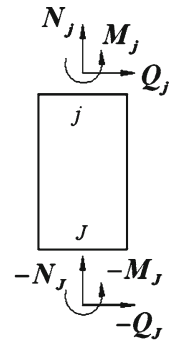

Fig. 3 A GB element composed of an aggregate beam, an interface beam and a matrix beam: a the GB element, $\mathbf{b}$ the aggregate beam, $\mathbf{c}$ the interface beam, $\mathbf{d}$ the matrix beam

$\mathbf{K}=\left[\begin{array}{cc}\mathbf{M}_{\mathbf{I}}^{\mathbf{a}}+\mathbf{M}_{I I}^{\mathrm{a}} \mathbf{R}_{\mathbf{I}} & \mathbf{M}_{\mathbf{I I}}^{\mathrm{a}} \mathbf{R}_{\mathbf{I I}} \\ \mathbf{S Y M} & \left(\mathbf{M}_{\mathbf{I I}}^{\mathrm{m}}\right)^{\mathrm{T}} \mathbf{R}_{\mathbf{I I I}}+\mathbf{M}_{\mathbf{I I I}}^{\mathbf{m}}\end{array}\right]$

where, the superscript of $\mathbf{M}$ declares the material property. In other words, $\mathbf{M}^{\mathbf{m}}, \mathbf{M}^{\mathbf{a}}$ and $\mathbf{M}^{\mathbf{i}}$ are, respectively, the stiffness matrix of matrix, aggregate and interface beams. $\mathbf{M}_{\mathbf{I}}^{\mathrm{a}}, \mathbf{M}_{\mathrm{II}}^{\mathrm{a}}$ and $\mathbf{M}_{\text {III }}^{\mathrm{a}}$ denote the top-left, top-right and bottom-right $3 \times 3$ sub-matrix of $\mathbf{M}^{\mathbf{a}}$, respectively, and the same notation rule is also used to $\mathbf{M}^{\mathbf{i}}$ and $\mathbf{M}^{\mathbf{m}}$. $\mathbf{R}_{\mathbf{I}}, \mathbf{R}_{\mathbf{I I}}$ and $\mathbf{R}_{\mathbf{I I I}}$, respectively, denote the top-left, top-right, bottom-right $3 \times 3$ sub-matrix of $\mathbf{R}$.

It can be proved that the matrix $\mathbf{K}$ generally has the following distribution of non-zero elements:

$$
\mathbf{K}=\left[\begin{array}{cccccc}
\mathrm{K}_{11} & 0 & -\mathrm{K}_{34} & -\mathrm{K}_{11} & 0 & -\mathrm{K}_{46} \\
& \mathrm{~K}_{22} & 0 & 0 & -\mathrm{K}_{22} & 0 \\
& & \mathrm{~K}_{33} & \mathrm{~K}_{34} & 0 & \mathrm{~K}_{36} \\
& & & \mathrm{~K}_{11} & 0 & \mathrm{~K}_{46} \\
& & \mathrm{SYM} & & \mathrm{K}_{22} & 0 \\
& & & & & \mathrm{~K}_{66}
\end{array}\right] .
$$

It can be found that $\mathbf{K}$ in Eq. (10) has more independent elements than $\mathbf{M}$ in Eq. (1). For the sake of convenience, the following matrix $\mathbf{K}_{\mathrm{N}}$ is defined as

$$
\mathbf{K}_{\mathrm{N}}=\left[\begin{array}{cccccc}
0 & 0 & 0 & 0 & 0 & 0 \\
& \mathrm{~K}_{22} & 0 & 0 & -\mathrm{K}_{22} & 0 \\
& & 0 & 0 & 0 & 0 \\
& & & 0 & 0 & 0 \\
& \mathrm{SYM} & & & \mathrm{K}_{22} & 0 \\
& & & & & 0
\end{array}\right] .
$$

As discussed in the introduction, shrinkage-induced cracking is dominantly responsible for the occurrence of the weakest interfacial phase. Therefore, it is more reasonable to take the investigated composites as composites with a much higher interface strength than the value used by van Mier et al. [30] and Liu et al. [20], and we do not distinguish interface from matrix any more in the current investigation. Furthermore, any multi-phase element shown as Fig. 3 is excluded 
(a)

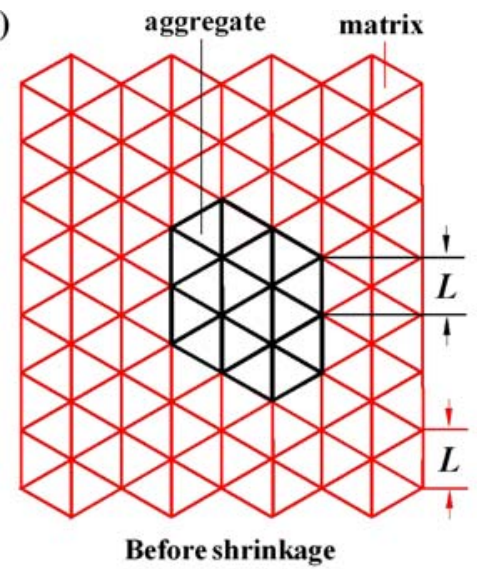

(b)
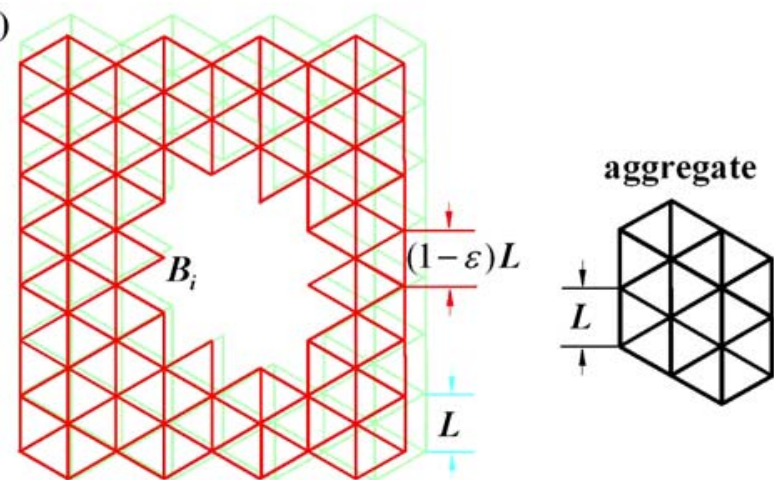

Matrix shrinkage rate $=\varepsilon$

Fig. 4 Sketch map of shrinkage-induced mismatch: a initial state before shrinkage, $\mathbf{b}$ size change of matrix lattice due to its shrinkage

in order to simplify the programming task. As a result, the lattice used in the current investigation is shown as Fig. 4a instead of Fig. 1a. Obviously, the classical beam-lattice used by Karihaloo et al. [13] or van Mier et al. [30] is an alternative equivalent to the GB lattice in that case.

\section{New method to simulate mismatch deformation due to matrix uniform shrinkage}

Nearly every numerical technique for simulating failure behavior in heterogeneous brittle media can be divided into two kinds of operations: one is solution of a proper linear elastic problem, and the other is detection of critical elements. When it comes to shrinkage failure processes, the corresponding linear elastic problem, i.e. the mismatch deformation cannot be solved by directly using the classical finite element method, because no obvious external mechanical loads exist as discussed in the introduction.

As a solution, several simplifications and assumptions are firstly made and then a new method is proposed here to obtain the mismatch displacement field.

In principle, drying of cement-based composites is multiphysics and even coupled with chemical reactions. The characteristic time is usually $\sim$ month. Therefore, a direct simulation seems to be a very hard task. However, different physical phenomena such as moisture transport and temperature change always play there influences in shrinkageinduced cracking by increasing the mismatch degree between deformations of matrix and aggregates. In other words, cracking is induced directly by mismatch deformation instead of moisture and temperature. As a result, when the main research interest is shrinkage-induced spontaneous cracking, it seems acceptable to take the shrinkage-induced cracking as a pure mechanical problem, i.e. progressive cracking driven by development of mismatch deformation.

To further simplify the analysis, the following approximations are adopted:

1. Small mismatch deformation. The change of the element size due to shrinkage is infinitely small when compared with the size before shrinkage.

2. Constant material properties. In principle, mechanical properties of matrix-phase change with shrinkage, which is neglected in the current study.

3. Uniform shrinkage is assumed in the matrix, while no shrinkage happens in aggregates.

Under assumption 1, the element stiffness matrix can be calculated based on the initial non-shrinkage configuration throughout the shrinkage process. Therefore, with the help of assumption 2, the stiffness matrix keeps unchanged unless the element fails.

At last, the problem becomes a pure linear elastic one. Then a numerical algorithm is introduced to solve it. Without loss of generality, we take the specimen shown in Fig. 4 as an example. A aggregate is embedded in the matrix. Before shrinkage, both the aggregate-phase element and the matrixphase element has the common length $L$, therefore, there is no interaction between the aggregate and matrix (Fig. 4a). After shrinkage, as shown in Fig. 4b, the length of the matrixphase element becomes $(1-\varepsilon) L(0<\varepsilon<<1)$, while the aggregate-phase element keeps the length of $L$. Here, $\varepsilon$ is called the shrinkage rate. When the aggregate lattice-structure is embedded into the shrunken matrix lattice, non-zero interaction appears between them because the matrix "hole" cannot accommodate the aggregate bigger than it without deforming. At the same time, mismatch deformation arises. The source of the mismatch phenomenon is being discussed in more detail later in this section.

The mismatch deformation seems hard to solved by directly using the classical finite element method. This difficulty is overcome by a new method as follows: the actual mismatch deformation field is decomposed into two virtual fields, both of which can be obtained by the classical FEM. Then the actual response is obtained by adding together the two virtual displacement fields. 
(a) D

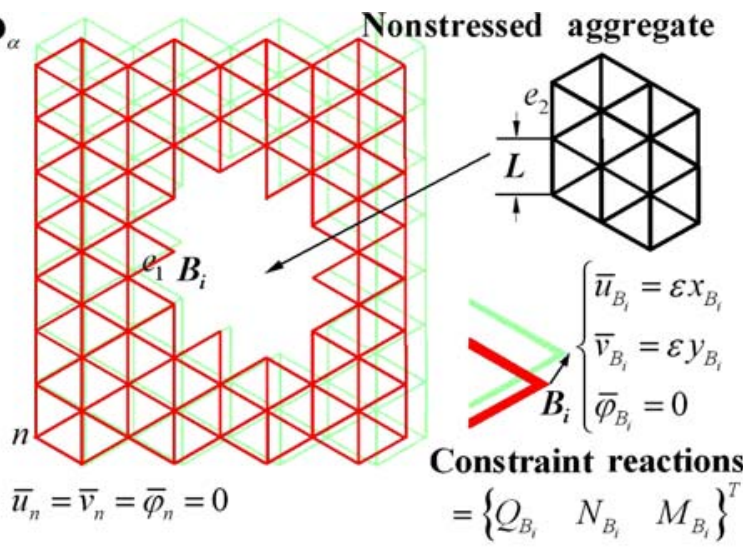

(b) D

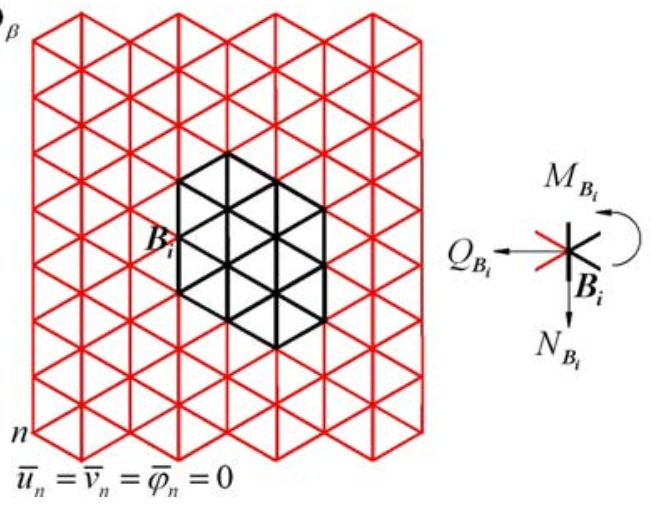

Fig. 5 Sketch map of calculating the two virtual displacement fields a $\mathbf{D}_{\alpha}, \mathbf{b} \mathbf{D}_{\beta}$

Just before explaining the two virtual displacement fields, a naming assumpsit is made: the matrix part of the specimen shown as Fig. 4b is called "matrix specimen". The distributed nodes on the boundary between matrix and aggregate are denoted as $\left\{B_{i} \mid i=1,2, \ldots, 10\right\}$.

- The first virtual displacement field $\mathbf{D}_{\alpha}$. The aim is to produce a displacement field in which the aggregate is in a non-stressed state. The investigated object is the matrix specimen. On its internal boundary $\left\{B_{i} \mid i=1,2, \ldots, 10\right\}$, the following boundary conditions are applied (Fig. 5a)

$$
\begin{gathered}
\bar{u}_{B_{i}}=\varepsilon x_{B_{i}}, \quad \bar{v}_{B_{i}}=\varepsilon y_{B_{i}}, \quad \bar{\varphi}_{B_{i}}=0, \\
B_{i} \in\left\{B_{i} \mid i=1,2, \ldots, 10\right\}
\end{gathered}
$$

where $\left(x_{B_{i}}, y_{B_{i}}\right)$ is the coordinate of node $B_{i}$.

And at some matrix node $n$, both translation and rotation are not permitted, which will be explained later:

$\bar{u}_{n}=\bar{v}_{n}=\bar{\varphi}_{n}=0$.

How to choose the proper node $n$ will be discussed later.

Then solve the above boundary value problem, and obtain the displacement field of the matrix specimen. On the boun- dary $\left\{B_{i} \mid i=1,2, \ldots, 10\right\}$, constraint reactions are calculated node by node, and denote the distributed constraint reaction system as $\left\{\left\{Q_{B_{i}} N_{B_{i}} M_{B_{i}}\right\}^{\mathrm{T}} \mid i=1,2, \ldots, 10\right\}$. Obviously, displacement boundary conditions in Eq. (12) are totally equivalent to the force boundary condition as follows:

$\bar{Q}_{B_{i}}=-Q_{B_{i}}, \quad \bar{N}_{B_{i}}=-N_{B_{i}}$,

$\bar{M}_{B_{i}}=-M_{B_{i}} \quad B_{i} \in\left\{B_{i} \mid i=1,2, \ldots, 10\right\}$.

Namely applying the force system $\left\{\left\{-Q_{B_{i}}-N_{B_{i}}-M_{B_{i}}\right\}^{\mathrm{T}}\right.$ $\mid i=1,2, \ldots, 10\}$ on to the internal boundary nodes.

It is notable that the internal boundary of the matrix specimen is extended under conditions in Eq. (12) to a degree that the aggregate can just be embedded into the matrix hole without interacting with the deformed matrix specimen. Then put the non-stressed aggregate into the deformed matrix hole, and note the displacement field of the total specimen as $\mathbf{D}_{\alpha}$.

The displacement field $\mathbf{D}_{\alpha}$ has a mismatch characteristic: the aggregate is in a non-stressed state, but the matrix specimen is deformed under the boundary conditions in Eqs. (12) and (13). The source of the mismatch lies in the difference between non-stressed reference configurations of the matrix specimen and the aggregate: the displacement of the matrix specimen is measured referring to the shrunken matrix lattice with element length $(1-\varepsilon) L$, but that of the aggregate is measured referring to the aggregate lattice with element length $L$.

As a direct result of this kind of mismatch in $\mathbf{D}_{\alpha}$ (Fig. 5a), the displacement of each node in $\left\{B_{i} \mid i=1,2, \ldots, 10\right\}$ has two values at the same time. When considering the deformation in some matrix element including node $B_{i}$, for example element $e_{1}$ in Fig. 5a, the displacement of $B_{i}$ should be set to $\bar{u}_{B_{i}}=\varepsilon x_{B_{i}}, \bar{v}_{B_{i}}=\varepsilon y_{B_{i}}, \bar{\varphi}_{B_{i}}=0$, i.e. the same value as Eq. (12). However, when considering the deformation of an aggregate element including $B_{i}$, for example element $e_{2}$ in Fig. 5a, the displacement should be zero at node $B_{i}$. In fact, the whole aggregate does not deform and displace in displacement $\mathbf{D}_{\alpha}$. Both of the two sets of displacement values for $\left\{B_{i} \mid i=1,2, \ldots, 10\right\}$ should be noted down for further analysis.

- The second virtual displacement field $\mathbf{D}_{\beta}$. The investigated object is the specimen shown in Fig. 5b. At the matrix node $n, \bar{u}_{n}=\bar{v}_{n}=\bar{\varphi}_{n}=0$ [Eq. (13)]. On the boundary between matrix and aggregate, i.e. $\left\{B_{i} \mid i=1,2, \ldots, 10\right\}$, apply the force system $\left\{\left\{Q_{B_{i}} N_{B_{i}} M_{B_{i}}\right\}^{\mathrm{T}} \mid i=1,2, \ldots\right.$, $10\}$ mentioned above on to the corresponding nodes

$$
\begin{aligned}
\bar{Q}_{B_{i}} & =Q_{B_{i}}, \quad \bar{N}_{B_{i}}=N_{B_{i}}, \\
\bar{M}_{B_{i}} & =M_{B_{i}} \quad B_{i} \in\left\{B_{i} \mid i=1,2, \ldots, 10 .\right\}
\end{aligned}
$$

Under this condition, both matrix and aggregate will deform together. The corresponding displacement field is 
obtained by conducting a linear elastic analysis and called $\mathbf{D}_{\beta}$. Note that $\mathbf{D}_{\beta}$ is an ordinary field without mismatch.

At last, the mismatch displacement field $\mathbf{D}$ can be expressed in the form

$\mathbf{D}=\mathbf{D}_{\alpha}+\mathbf{D}_{\beta}$

which can be explained as follows: $\mathbf{D}_{\alpha}$ can be taken as the displacement field by applying the external force system $\left\{\left\{-Q_{B_{i}}-N_{B_{i}}-M_{B_{i}}\right\}^{\mathrm{T}} \mid i=1,2, \ldots, 10\right\}$, and $\mathbf{D}_{\beta}$ is the displacement under action of the external force system $\left\{\left\{Q_{B_{i}} N_{B_{i}} M_{B_{i}}\right\}^{\mathrm{T}} \mid i=1,2, \ldots, 10\right\}$. Therefore, $\mathbf{D}=\mathbf{D}_{\alpha}+$ $\mathbf{D}_{\beta}$ is the displacement field when the external load is zero with the help of the superposition principle [6]. The difference between non-stressed reference configurations which is the resource of mismatch has been considered when measuring displacements of matrix and aggregate in $\mathbf{D}_{\alpha}$. In other words, $\mathbf{D}_{\alpha}$ is a mismatch field. So this mismatch should not be considered again when calculating $\mathbf{D}_{\beta}$, resulting that $\mathbf{D}_{\beta}$ is continuous (non-mismatched). Again, nodes in $\left\{B_{i} \mid i=1,2, \ldots, 10\right\}$ also have two sets of displacement values in $\mathbf{D}=\mathbf{D}_{\alpha}+\mathbf{D}_{\beta}$, like that in $\mathbf{D}_{\alpha}$ discussed above.

By the way, boundary conditions in Eq. (13) at node $n$ are necessary for calculating $\mathbf{D}_{\alpha}$ and $\mathbf{D}_{\beta}$ (Fig. 5). Otherwise, $\mathbf{D}_{\beta}$ cannot be calculated correctly because the problem shown in Fig. 5b becomes statically indeterminate. Nevertheless, the final actual displacement field $\mathbf{D}=\mathbf{D}_{\alpha}+\mathbf{D}_{\beta}$ is independent of the position of node $n$ because there is only a rigid displacement delta between fields by choosing different node $n$. Therefore, boundary conditions in Eq. (13) can be applied on an arbitrary node in the matrix region except the internal boundary nodes $\left\{B_{i} \mid i=1,2, \ldots, 10\right\}$.

In a word, Eq. (16) represents the basic idea of the current investigation to simulate mismatch deformation due to matrix shrinkage in cement-based composites.

\section{Global numerical procedure}

Fracture processes under external loads are usually simulated by successive appearances of critical elements. So is the shrinkage-induced fracture, except that external loads are replaced by the shrinkage rate. A complete failure process can be obtained by carrying out the following steps sequentially:

Step 1: Add the shrinkage rate as a trial value $\varepsilon$.

Step 2: Perform an analysis by means of the method introduced in Sect. 3, and obtain the mismatch displacement field $\mathbf{D}=\mathbf{D}_{\alpha}+\mathbf{D}_{\beta}$.

Step 3: Extract the critical element from the results by using the "event-by-event" algorithm which will be introduced in the following Sect. 4.2.

Step 4: Calculate the critical shrinkage rate as the trial value times the current strength divided by the correspon- ding stress of the critical element. Note down the critical displacement field.

Step 5: Adjust the stiffness and strength of the critical element.

Step 6: Keep the critical shrinkage rate unchanged, release the corresponding internal forces of the critical element(s), and perform a new linear elastic analysis to check if another element will fail.

Step 7: Repeat Step 6 until no more element fails, then note down the final displacement field.

Step 8: Repeat Steps 1-7 until the complete failure of the specimen.

Step 9: Observe and analyze the result.

To make the above chart more clear, it is necessary to introduce the failure criterion and the algorithm to detect critical element(s), both of which have been discussed in more detail in Liu et al. [20].

\subsection{Mohr-Coulomb criterion}

Mohr-Coulomb theory is a mathematical model describing the response of materials such as rock, rubble piles or concrete to shear stress as well as normal stress. Most of the classical engineering materials somehow follow this rule in at least a portion of their shear failure envelope. The criterion can be expressed in the form

$|\tau|<c-\sigma \tan \phi$

where, $c$ is the cohesive strength and $\phi$ is the friction angle; $\tau$ and $\sigma$ are the shear stress and the normal stress, respectively.

Because materials such as concrete/rock have a very low tensile strength $f_{t}$ as compared with their compressive strength and shear strength, the Mohr-Coulomb strength surface with tension cut-off [2] is adopted here (Fig. 6). Concrete's compressive strength $f_{c}$ is generally 10-20 times the value of its tensile strength, so $f_{c}=10 f_{t}$ is used in this paper. Furthermore, the failure surface can be expressed by the following three inequations:

$$
\begin{aligned}
& \sigma<f_{t} \\
& |\tau|<c-\sigma \tan \phi \\
& \sigma>-f_{c} .
\end{aligned}
$$

Then the implementation of the Mohr-Coulomb criterion shown in Fig. 6 is introduced. It is notable that we find out the critical element by checking the stresses in beams of GB elements. The normal stress can be expressed in the form [30]

$\sigma=\frac{N}{A}+\alpha \frac{\left(\left|M_{i}\right|,\left|M_{j}\right|\right)_{\max }}{W}$

where, $N$ is the normal force in the considered beam, $M_{i}$ and $M_{j}$ are the bending moments at the nodes $i$ and $j$ of the beam, 


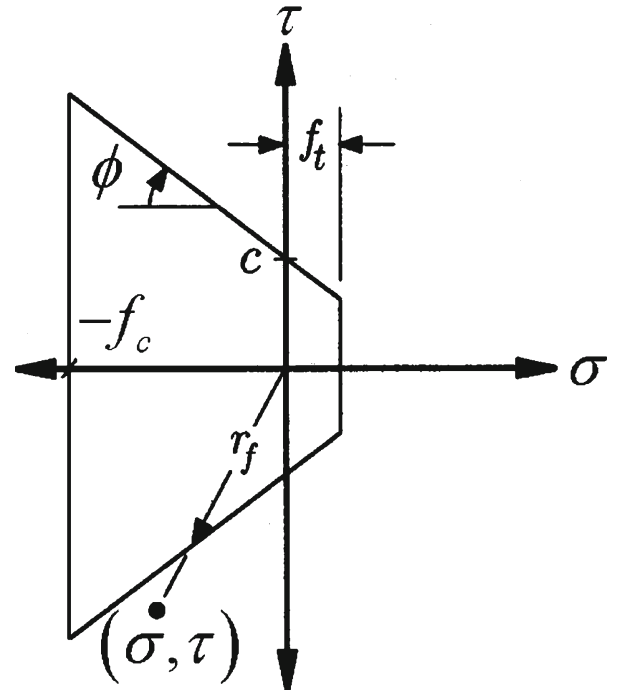

Fig. 6 Mohr-Coulomb strength surface with tension cut-off

and $W=t^{(b)} l^{2} / 6$ is the section modulus. The coefficient $\alpha$ regulates what part of the bending moment is considered. Lilliu and van Mier [17] have shown that simulation results are also satisfactory in the case of $\alpha=0$ though $\alpha$ is usually set to $0.005[13,18]$. Therefore, $\alpha$ is also set to zero in this paper. Another advantage of $\alpha$ being zero lies in that three beams in every GB element have the uniform normal stress because both $N$ and $A$ are uniform throughout every GB element.

From Eq. (1), the shear force can be expressed in the form

$Q_{1}=-Q_{2}=M_{11}\left(u_{1}-u_{2}\right)-M_{34}\left(\varphi_{1}+\varphi_{2}\right)$.

In consideration of the equilibrium conditions at $I$ and $J$ (Fig. 3), it can be found that the shear force is also uniform throughout the GB element. Therefore, the shear stress can be calculated as

$|\tau|=\frac{\left|Q_{1}\right|}{A}=\frac{1}{A}\left|M_{11}\left(u_{1}-u_{2}\right)-M_{34}\left(\varphi_{1}+\varphi_{2}\right)\right|$.

Actually, in order to judge if a GB element becomes critical, it is not necessary to check all its three beams. Only its middle beam needs to be checked instead mainly because both $\sigma$ and $\tau$ are uniform in every GB element, which has been discussed above. Furthermore, it makes the following assumption reasonable: when some GB element becomes critical, it cracks into two fragments of the same span, i.e. $L / 2$, where $L$ is the length of the GB element. Therefore, if all six GB elements starting from node $i$ have failed, the isolated material domain around node $i$, called influence zone of node $i$ has the geometry shown as Fig. 7a. The GB lattice can be also taken as a network by gluing a large amount of this kind of material domains, shown as Fig. 7b, where the short line-segment crossing the middle of every GB element indicates the potential crack path.

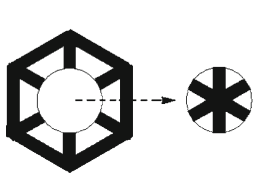

(a)

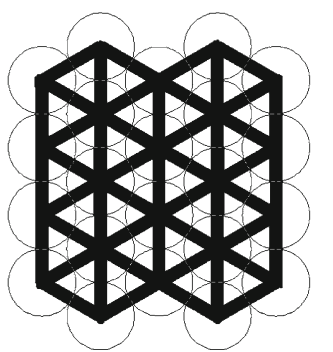

(b)

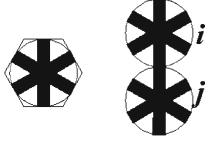

(c)
Fig. 7 A GB lattice with circular nodal influence zones: a a single nodal influence zone isolated be surrounding failures, $\mathbf{b}$ potential failure positions of the lattice, $\mathbf{c}$ comparison of circular and hexagon influence zones, $\mathbf{d}$ sketch-map of contact

Then an important approximation is made: the material domain around every node is circular and its diameter is $L$, which is the same as the bonded-particle model (BPM) [24]. When calibrating the GB lattice [18], it is assumed that the influence zone (unit cell) is hexagonal, which is $\pi / 2 \sqrt{3}$ times the area of the circle (Fig. 7c). This difference can be avoided easily when using the equivalence of strain energy between the GB lattice and its continuum counterpart. But this difference is neglected in this paper. On one hand, it doesn't influence the results seriously. On the other hand, even though the model is a very rough approximation of reality, this appears throughout all kinds of lattice-type approaches.

When the two crack surfaces of the former cracked element contact each other, the contact analysis between crack surfaces becomes necessary. In this paper, we only study the simplest case-smooth contact. Therefore, the contact element can only bear compressive actions along the direction of element $i j$, and the stiffness matrix can be expressed in the form

$\mathbf{K}_{\text {contact }}=(1-D) \mathbf{K}_{N}$

where, $D$ is the damage factor due to the deformation history. Of course, the concept of the damage factor here is extremely rough as compared with the one in the classical damage mechanics which increases gradually with external loads [16]. However, $D$ in Eq. (22) also indicates the degradation of material properties, so basically has the same physical meaning of the classical damage factor [14].

In this paper, it is assumed that the failure modes violating different inequations in Ineqs. (18) may generally produce different damage factors. In the following of this section, the non-linear algorithm is introduced for that general case.

\subsection{Event-by-event algorithm}

Fracture is simulated by successive occurrences of "events", which may be failure of critical intact or partly failed elements, 
or contact/separation of former cracked elements. Then, the essential of numerical simulation is to detect new event(s) correctly. The event-by-event method is adopted here [2,25]. The magnitude of deformation change of the specimen in every step depends on the appearance of new critical element(s). After the current displacement field has changed, the normal stress $\sigma$ and the shear stress $\tau$ acting in each beam are compared with the fracture surface, the criterion for fracture is

$R=\frac{r}{r_{f}}>1$

where $r=\left(\sigma^{2}+\tau^{2}\right)^{0.5}$ and $r_{f}$ is as defined in Fig. 6. Analogously, the criterion for separation/contact of cracked elements is

$R=\frac{\sqrt{(\Delta u)^{2}+(L+\Delta v)^{2}}}{L}>1($ contact $\rightarrow$ separation $)$

$R=\frac{L}{\sqrt{(\Delta u)^{2}+(L+\Delta v)^{2}}}>1($ separation $\rightarrow$ contact $)$

where, $\Delta u=u_{j}-u_{i}$ and $\Delta v=v_{j}-v_{i}$ are relative displacements between nodes $i$ and $j$ in element $i j$ shown in Fig. 7.

Only one event is permitted per computational cycle. If several elements have $R>1$, the element with the highest $R>1$ undergoes new event. As for the critical element, the update of stiffness matrix and the release of internal forces are introduced as follows:

(1) If Ineq. (18a) is violated, $\left\{\begin{array}{llllll}Q_{i} & N_{i} & M_{i} & Q_{j} & N_{j} & M_{j}\end{array}\right\}^{\mathrm{T}}$ is released, and the element stiffness matrix $\mathbf{K}$ is set to zero. It is notable that this element is definitely under tensile state in the current step. However, this element is possible to recur due to contact in the following steps. Once contact happens, the recurred stiffness matrix is

$$
\stackrel{\mathrm{T}}{\mathbf{K}}=(1-\stackrel{\mathrm{T}}{D}) \mathbf{K}_{\mathrm{N}}
$$

where, the superscript " $\mathrm{T}$ " in $\stackrel{\mathrm{T}}{\mathbf{K}}$ and $\stackrel{\mathrm{T}}{\mathrm{D}}$ represents "Tension". Thus, $\stackrel{\mathrm{T}}{D}$ denotes the damage factor due to the failure by violating Ineq. (18a), i.e. maximum tensile stress criterion. $\mathbf{K}_{\mathrm{N}}$ has been defined in Eq. (11).

(2) If Ineq. (18b) is violated, there are two possible cases. In one case, the normal stress $\sigma$ acting in the critical element is positive, i.e. tensile stress, so the element is not possible to become contact at the very beginning of stress redistribution. As a result, $\left\{\begin{array}{llll}Q_{i} & N_{i} & M_{i} & Q_{j}\end{array}\right.$ $\left.\begin{array}{ll}N_{j} & M_{j}\end{array}\right\}^{\mathrm{T}}$ is released, and the element stiffness matrix $\mathbf{K}$ is set to zero. In the other case, $\sigma$ is negative, i.e. compressive stress, so the critical element becomes contacting once violation happens. Then, $\left\{\begin{array}{lll}Q_{i} & \stackrel{\mathrm{MC}}{D} N_{i} & M_{i}\end{array}\right.$ $\left.Q_{j} \stackrel{\mathrm{MC}}{D} N_{j} \quad M_{j}\right\}^{\mathrm{T}}$ is released, and the stiffness matrix is updated as

$$
\stackrel{\mathrm{MC}}{\mathbf{K}}=(1-\stackrel{\mathrm{MC}}{D}) \mathbf{K}_{\mathrm{N}}
$$

where, the superscript "MC" stands for "MohrCoulomb". $\stackrel{\mathrm{MC}}{D}$ denotes the damage factor due to the failure under the actions of both the shear stress and the normal stress, defined as Mohr-Coulomb criterion in Ineq. (18b).

(3) Three kinds of elements are possible to violate Ineq. (18c): intact elements, elements damaged by violating Ineq. (18a) previously, and elements damaged by violating Ineq. (18b) previously. As for intact elements,

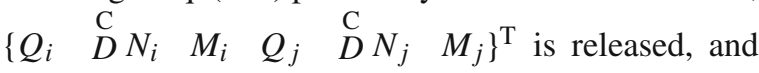
the stiffness matrix is updated as

$$
\stackrel{\mathrm{C}}{\mathbf{K}}=(1-\stackrel{\mathrm{C}}{D}) \mathbf{K}_{\mathrm{N}}
$$

where, the superscript "C" represents "Compression". As for the second kind of elements, $\left\{\begin{array}{ll}Q_{i} & (\mathrm{C} \\ D & -\mathrm{T} \\ D\end{array}\right) N_{i} /$ $\left.(1-\stackrel{\mathrm{T}}{D}) \quad M_{i} \quad Q_{j} \quad(\stackrel{\mathrm{C}}{D}-\stackrel{\mathrm{T}}{D}) N_{j} /(1-\stackrel{\mathrm{T}}{D}) \quad M_{j}\right\}^{\mathrm{T}} \quad$ is released, and the stiffness matrix is set to $\stackrel{\mathrm{K}}{\mathbf{K}}$. As for the third kind of elements, $\begin{cases}Q_{i} & (\stackrel{\mathrm{C}}{D}-\stackrel{\mathrm{MC}}{D}) N_{i} /(1-\stackrel{\mathrm{MC}}{D})\end{cases}$ $\left.M_{i} \quad Q_{j} \quad(\stackrel{\mathrm{C}}{D}-\stackrel{\mathrm{MC}}{D}) N_{j} /(1-\stackrel{\mathrm{MC}}{D}) \quad M_{j}\right\}^{\mathrm{T}}$ is released, and the stiffness matrix is also set to $\stackrel{\mathrm{K}}{\mathbf{K}}$.

\section{Examples of shrinkage-induced cracking}

Four cases are sequentially studied, called Cases $1-4$. Tests are performed on a GB lattice with a rectangular geometry of 9 by $66 \sqrt{3} / 5 \mathrm{~cm}$. The GB lattice employed has a total of 8,082 nodes and 23,861 elements. In Case 1, the micro-level material properties are shown in Table 1, where the difference from former parameter setting used [20,30] is that a bigger interface strength $2.5 \mathrm{MPa}$ instead of former 1.25 MPa is adopted in order to exclude influence of the shrinkage-induced cracking on the interface strength. About 17 hexagon aggregates with side length $6 L$ are randomly distributed, where $L$ is the element length. All elements are $\sqrt{3} / 10 \mathrm{~cm}$ long. The depth-to-span ratio is set to 1.0 for all beams. Uniform shrinkage is assumed in the matrix region, and aggregates do not shrinkage or expand spontaneously. 
Table 1 The micro-elastic and strength properties of phases

Case 2 is the same as Case 1 except that Young's modulus of aggregates is $1 / 4$ of the value in Case 1 , which represents a specimen including softer aggregates.

Case 3 is the same as Case 1 except that the side-length of hexagon aggregates is $2 L$ instead of $6 L$ in Case 1, i.e. specimen with smaller aggregates.

Case 4 includes more aggregates than Case 1: 22 aggregates with side length $6 L$ and 7 ones of $2 L$.

\subsection{Case 1: analyses of a typical shrinkage case}

All the cases have many common features even though their settings are different from each other. Therefore, Case 1, whose result is shown in Fig. 8, is chosen as a representative and analyzed in detail.

1. Differences can be found between shrinkage-induced cracking and failures under external loads such as uniaxial tensile experiment shown in Fig. 9. The process has a typical multi-crack characteristic. Shrinkage-induced cracks form a network-like shape all over the specimen. No obvious main crack passing through the specimen appears as generally happening in compressive/tensile experiments $[13,20,30]$. This is due to the difference between external load and "shrinkage load": The external load is usually applied in a fixed direction, and the internal force of the cracking element cannot be born by itself any more due to descending or vanishing of its carrying capacity. Therefore, this extra force is redistributed among the rest elements. In other words, the external load is endured by less and less elements, and failed elements put the nearby intact elements, especially ones on the tip of cracks, into a stress level easier to fail, but meanwhile unload the other regions of the specimen. While "shrinkage load" has not a fixed loading direction, leading to a more scattered crack pattern. A localized crack at one position cannot prevent appearance of cracks at other positions because no unloading mechanism in a fixed direction exists in the shrinkage process. In fact, surrounding neighborhood of every aggregate is potentially active to fail due to shrinkage-induced mismatch. Under the uniform shrinkage condition studied here, if the matrix specimen is taken as the investigated object, every aggregate acts as an "object applying force". From this viewpoint, loads are applied on two opposite edges in the tensile test, but "shrinkage load" is applied throughout the specimen and mismatch interactions arise around all internal boundaries between matrix and aggregates.

As a result of absence of main $\operatorname{crack}(s)$, no steep change of failed element number exists in the curve of Fig. 8a. In tensile/compression tests, a steep drop of load occurs usually directly following the peak load $[17,18]$, corresponding to formation of main $\operatorname{crack}(\mathrm{s})$ and steep increase of failed elements, shown as Fig. 9.

Compared Fig. 8a with Fig. 9a, the magnitude of failure number in shrinkage is $\sim 1,000$, while that is only $\sim 100$ in tensile tests, which indicating deformation in tensile failure is much more localized than the shrinkage process.

2. Cracking happens most easily in narrow matrix regions between the specimen boundary and aggregates, shown as Fig. 8b-d. Figure 10a gives a typical example of such boundary cracking. The mismatch deformation around these aggregates is greatly influenced by the near free boundary, which makes the narrow regions mentioned above deform much more greatly than other regions around the same aggregate. In the mismatch deformation field, the aggregate close to specimen boundary has a tendency of being squeezed out of the specimen by breaking the narrow matrix region. During the shrinkage process, free surfaces of the internal crack that has appeared in the early stage also act as "free boundary" (Fig. 10b), therefore, the nearby aggregate seems to be squeezed towards the crack surface and one or more new cracks from the aggregate to the free crack surface will occur. This is also very different from failure experiment under external loads where the crack appearing previously usually unloads the neighborhood of its two crack, leading this region does not fail any more.

From Figs. 8b-d and 10a, it can be found that these localized cracks near specimen boundary do not propagate further into inside once stopping at the aggregate surface. Obviously, this phenomenon must be reasonable because most concrete buildings whose surface covered with micro- and even macro-shrinkage induced cracks are still safe instead of collapse. 
Fig. 8 In case 1 , a the cumulative number of cracked elements versus displacement; and crack patterns at three levels: b level $a$; c level $b$; d level $c$

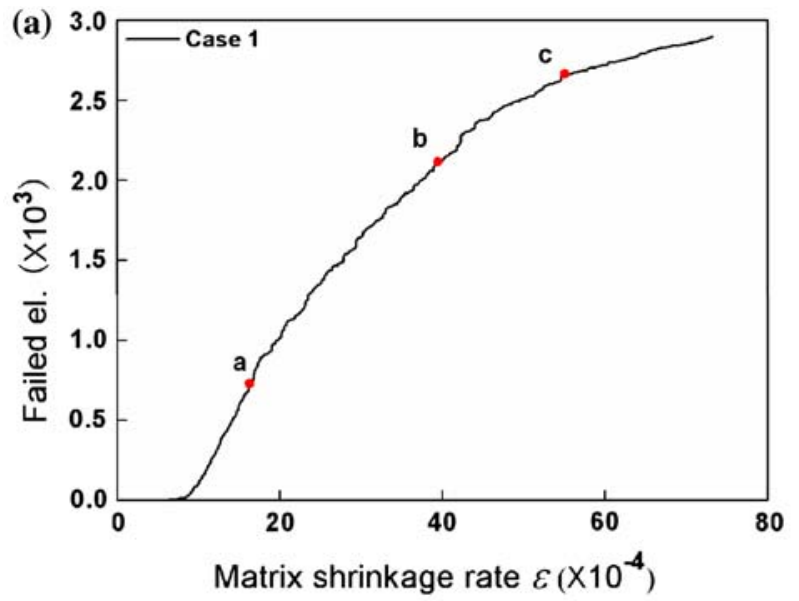

(b)

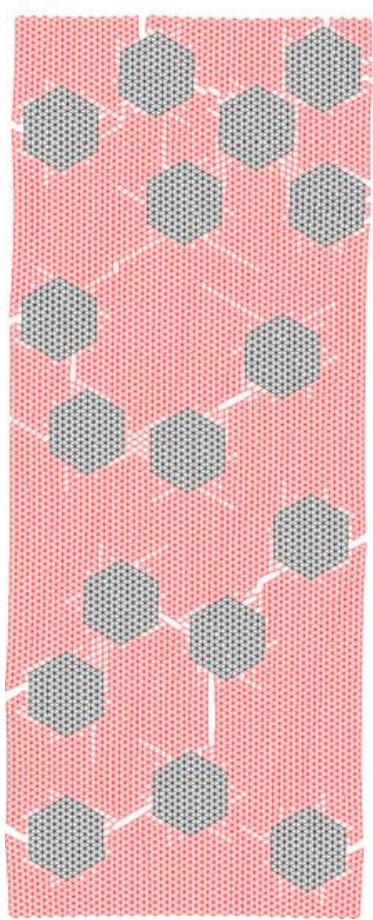

(c)

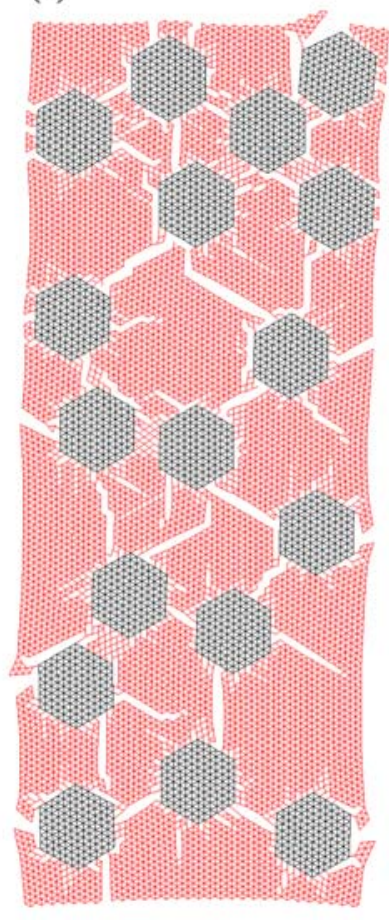

(d)

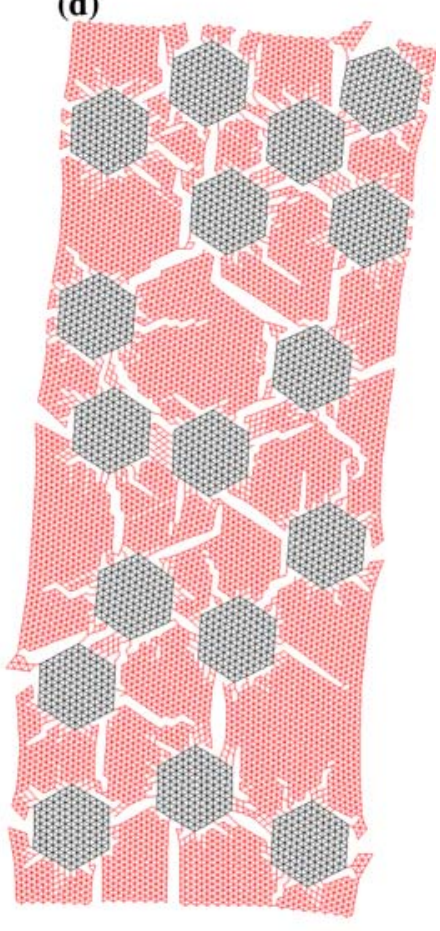

3. Elemental failures happen frequently around aggregates. These failures together with aggregates look like snowflakes. Majority of localized cracks start from aggregate surfaces, and center aggregates, resulting that matrix regions around aggregates are most damaged. Mismatch in displacement field arises in the boundary between matrix and aggregates, where deformation is much greater than other regions, therefore, elemental failures in matrix regions around aggregates happen much more easily.

4. Localized cracks have several common feathers. These cracks start from aggregates and stop propagating in matrix, specimen boundary or when blocked by another aggregate. Aggregates would not be split by the

arriving localized matrix cracks with the following reason: Aggregates are in a state of compression. The compressive action applied on aggregates is from matrix. The weaker matrix cannot of course compress the stronger aggregates into failure. Two opposite surface of every localized crack connecting two adjacent aggregates have arc-like shape caused by both matrix shrinkage and compressive actions from the two aggregates.

\subsection{Case 2: influence of aggregate Young's modulus}

Aggregates have a Young's modulus equal to $1 / 4$ of that in Case 1, indicating that aggregates in Case 2 are much softer. 
Fig. 9 A typical tensile result: a the dimensionless $P-\delta$ curve where load and displacement are divided by the values at the peak; $\mathbf{b}$ the cumulative number of cracked elements versus displacement; $\mathbf{c}$ crack pattern at level $a$
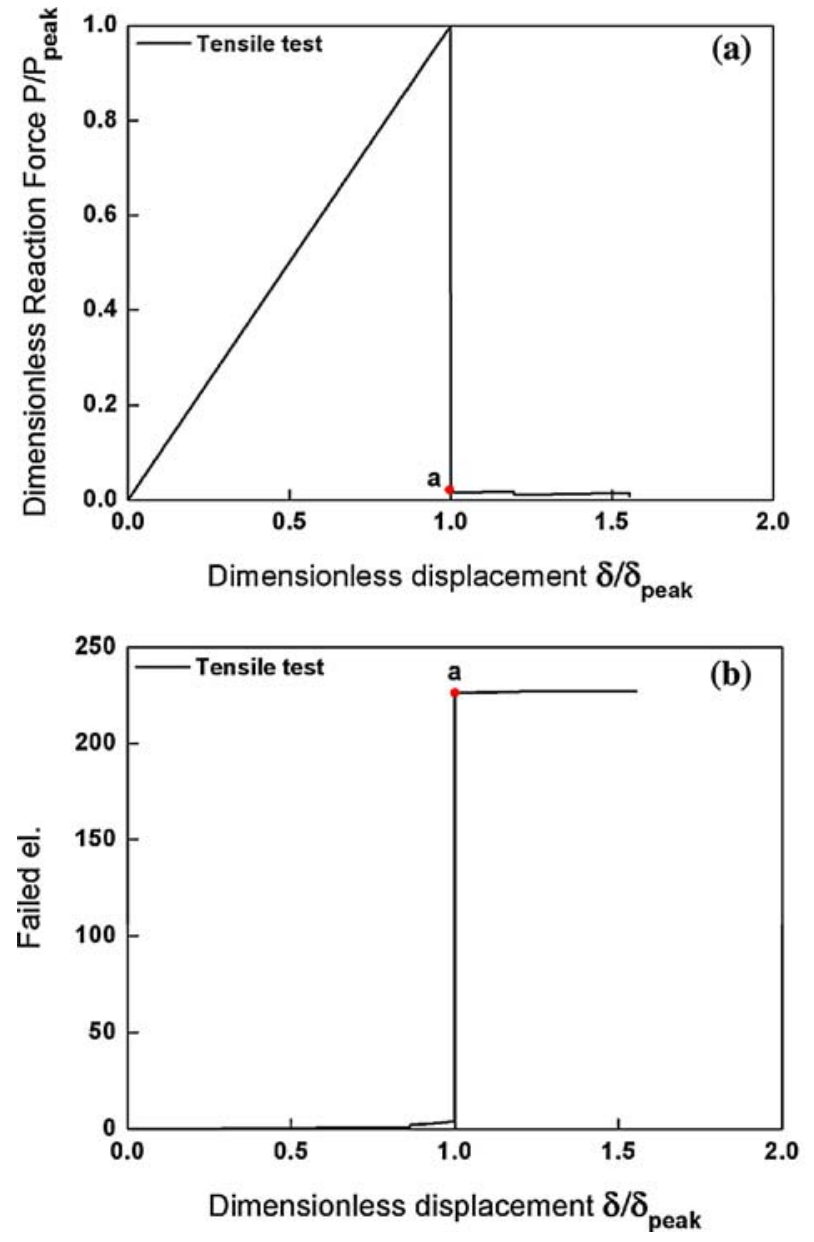

(c)

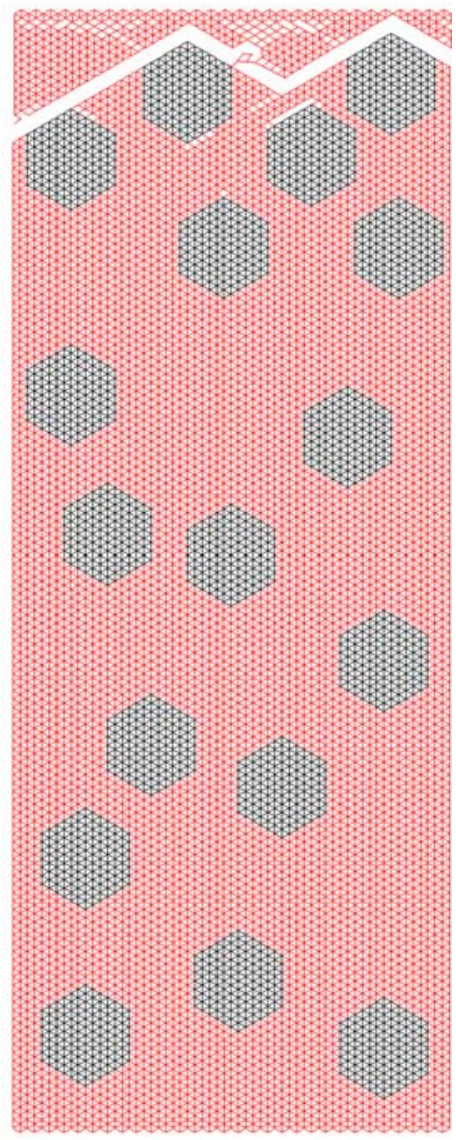

(a)
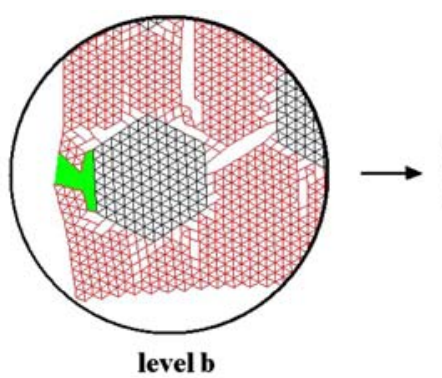

(b)

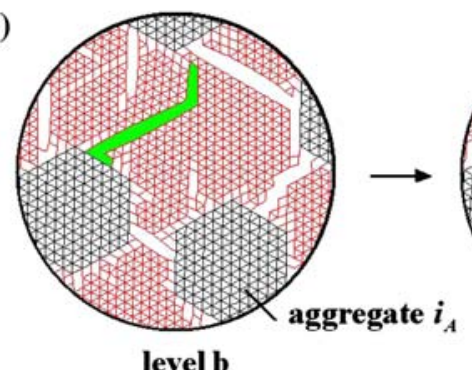

level b

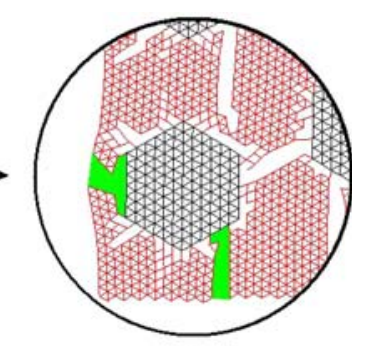

level c

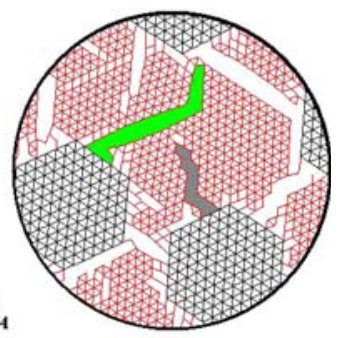

level c

Fig. 10 Development of local crack patterns in case 1: a cracks (in green) appears in the narrow matrix region between aggregate and specimen boundaries; $\mathbf{b}$ the gray crack arises when aggregate $i_{A}$ is squeezed towards the green crack (color available only in online publication)

Therefore, when the shrinkage rate is fixed, matrix has a smaller deformation in Case 2 than Case 1. In other words, failures happen less easily in Case 2 . Figure $11 \mathrm{~b}$ corresponding to $\varepsilon=24.87 \times 10^{-4}$ contains 579 failures, less than 712 in Fig. $8 \mathrm{~b}$ where the shrinkage is weaker, i.e. $\varepsilon=16.23 \times 10^{-4}$. This trend can be also found easily from Fig. 11a. When the total of failures is fixed as 2,100 , the shrinkage rate $\varepsilon$ has a value of $55.95 \times 10^{-4}$, much larger than $\varepsilon=39.2 \times 10^{-4}$ in Case 1. On the other hand, level $\mathrm{b}$ in Case 1 shown as Fig. $8 \mathrm{c}$ and level $\mathrm{b}$ in Case 2 shown as Fig. 11c have obviously distinguished crack patterns, even though exactly equal totals of failures and the same particle distribution. This suggests change of aggregate Young's modulus leads to a difference failure process. Figure 11d shows the crack pattern of late stage, where the specimen boundaries have been destroyed into separated sections and become obviously warped.

\subsection{Case 3: influence of aggregate size}

Each aggregate has the same center position as the correspondence in Case 1, but is much smaller. As mentioned in Sect. 3, all mismatch in deformation, i.e. the displacement 
Fig. 11 In case 2, a the cumulative number of cracked elements versus displacement; and crack patterns at three levels: b level $a$; c level $b$; d level $c$

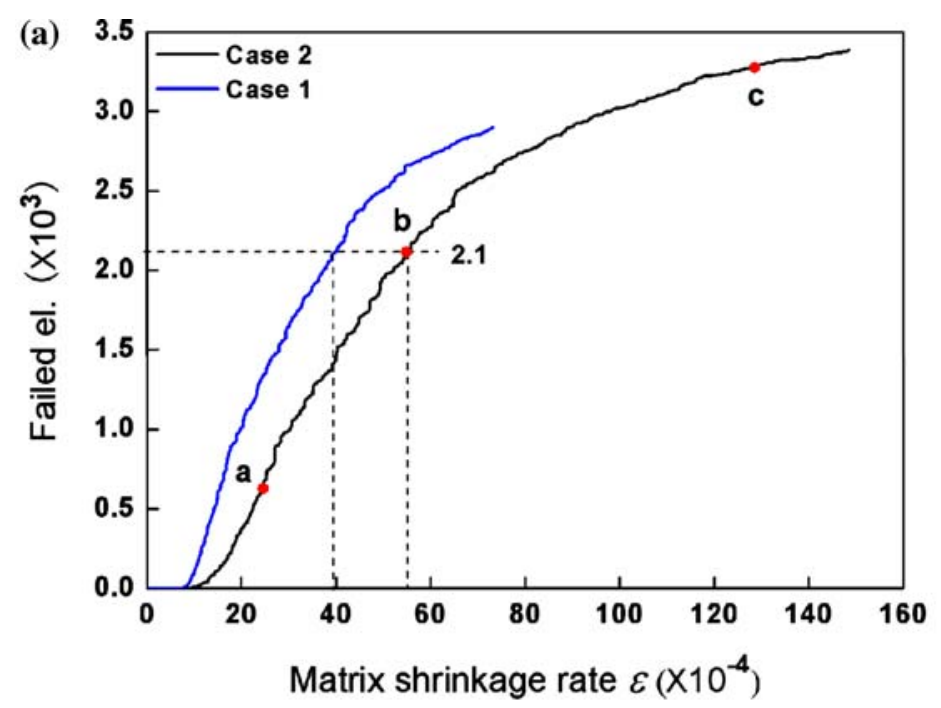

(b)

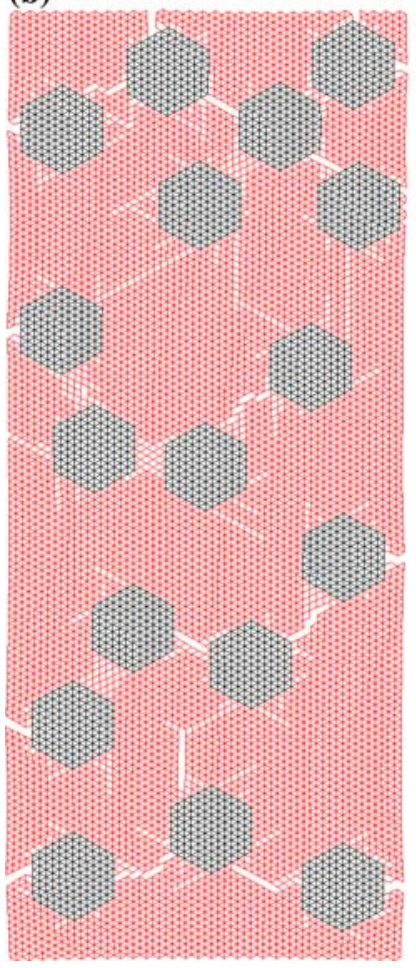

(c)

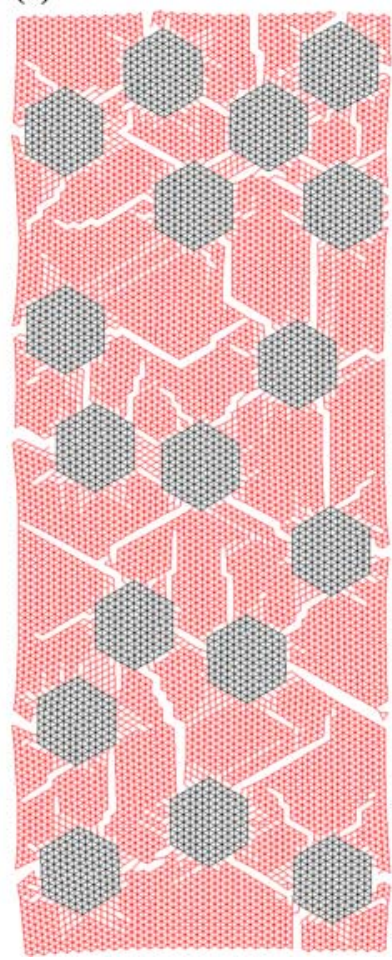

(d)

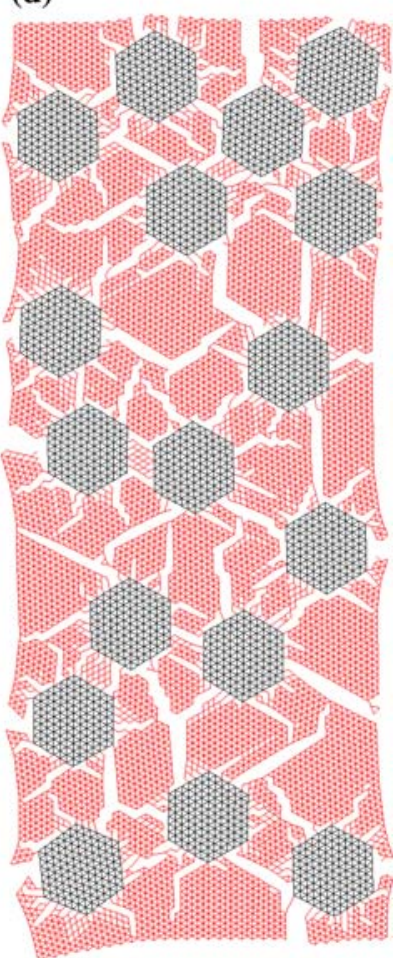

discontinuity happens on boundaries between matrix and aggregates. Additionally, the total length of these boundaries in a specimen is equal to the sum of all aggregates' perimeters. Therefore, the total length of mismatch lines in Case 3 is much smaller than Case 1. Furthermore, as mentioned in Case 1, as for the aggregate near to specimen boundary, matrix region in its neighborhood closest to boundary is very active to fail (Fig. 10a). Boundary of each aggregate is farer from the specimen free boundary in Case 3, leading to a lower failure possibility in the matrix regions between specimen boundary and aggregates. These reasons lead to a more gentle increase of failure total with shrinkage rate (Fig. 12a)
Due to these reasons, shrinkage-induced cracking happens less possibly than Case 1 when shrinkage rate is fixed. The shrinkage rate when the first elemental failure occurs is $14.66 \times 10^{-4}$, much larger than the corresponding value $6.40 \times 10^{-4}$ in Case 1. In micro-mechanics simulations of concrete, it is of course not very realistic to model every aggregate of any given size, and therefore, normally a lower cut-off is needed. Below the cut-off threshold aggregates are assumed to be part of matrix, where it is assumed that the matrix behaves like a continuum [30]. Obviously, the above trend of shrinkage-induced cracking with aggregate size observed can be taken as a positive evidence 
Fig. 12 In case 3, a the cumulative number of cracked elements versus displacement; and crack patterns at three levels: b level $a$; c level $b$; d level $c$

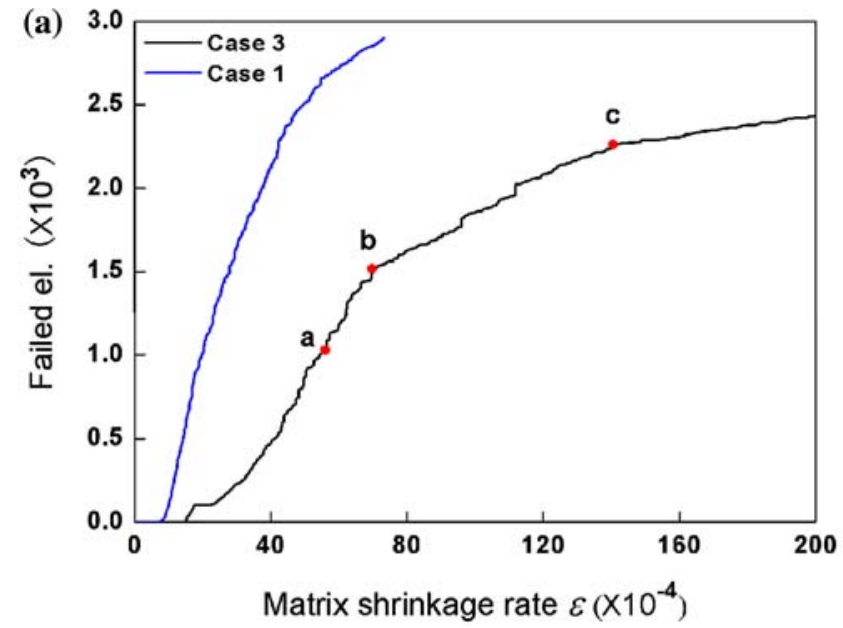

(b)

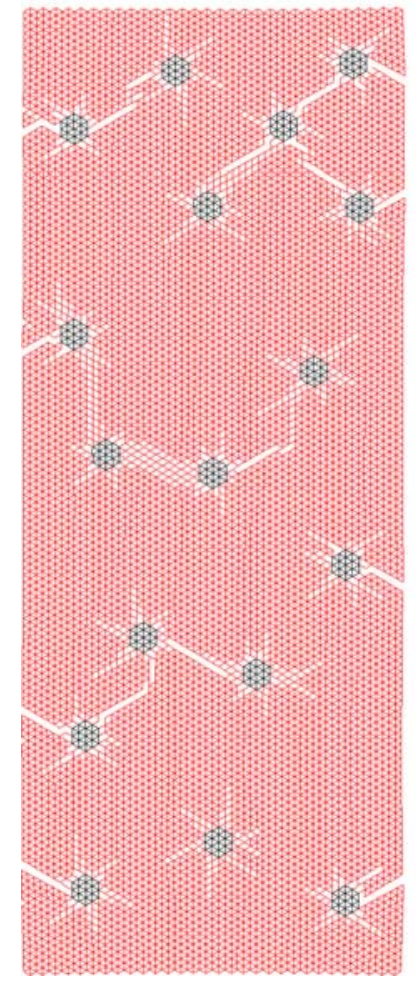

(c)

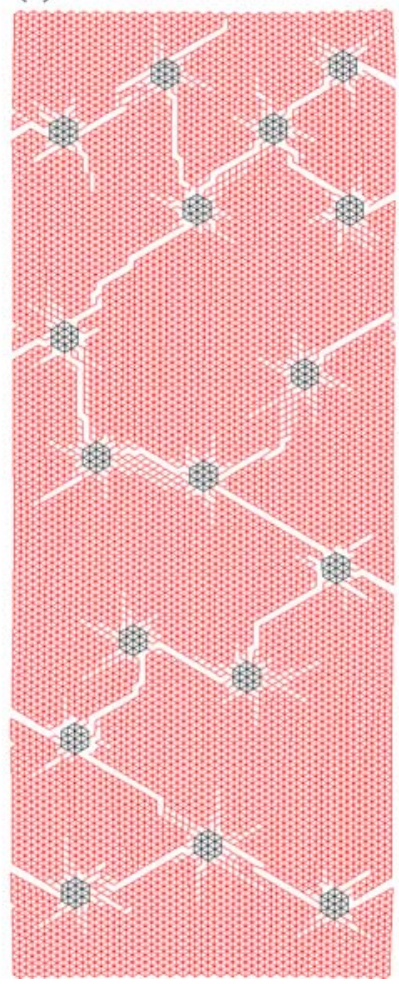

(d)

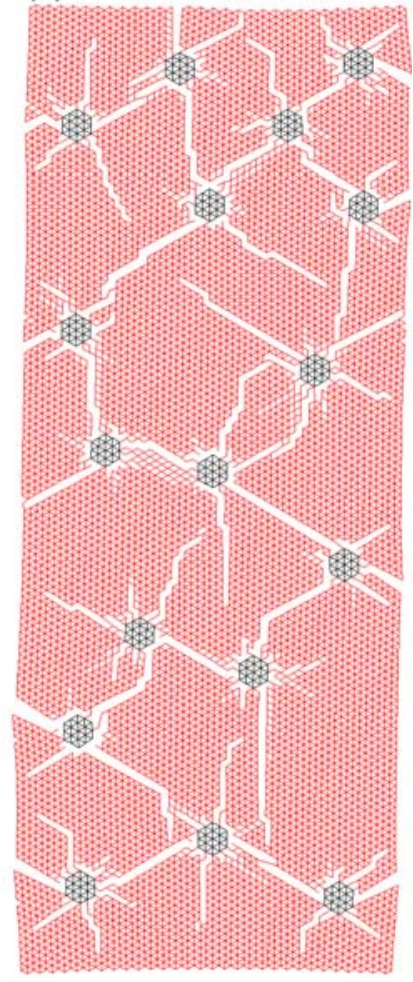

supporting this kind of cutting-off operation in aggregate size.

Deformation around smaller aggregate is influenced less seriously by specimen boundaries and other aggregates, leading to a more homogeneous deformation in different directions. Therefore, from Fig. 12b, a more obviously radiating feather of failure distribution can be found around every aggregate when compared with Fig. 8b.

There is a turning at level b in Fig. 12a: total of failures increases less steeply with shrinkage rate after level $b$ than before it. After level c, the increase becomes even more slowly. This can be explained as follows. As the shrinkage rate increases, more and more elements linking aggregates to matrix fail, resulting that aggregates become more isolated. Therefore, interactions between matrix and aggregates become weaker, or in other words the drive of cracking decreases with shrinkage rate. Obviously, the smaller aggregate becomes isolated more easily, and new localized cracks appear less possibly. Therefore, crack patterns shown in Fig. 12c, d looks less smashed than Fig. 8d.

\subsection{Case 4: influence of aggregate content}

Majority of local cracks except those between aggregates and specimen free boundaries start from one aggregate and end when arriving surface of another adjacent aggregate, as 


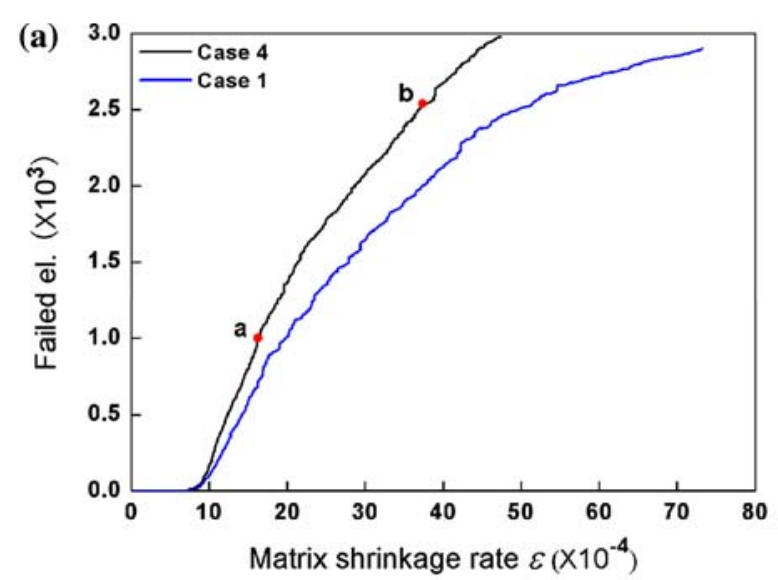

(b)

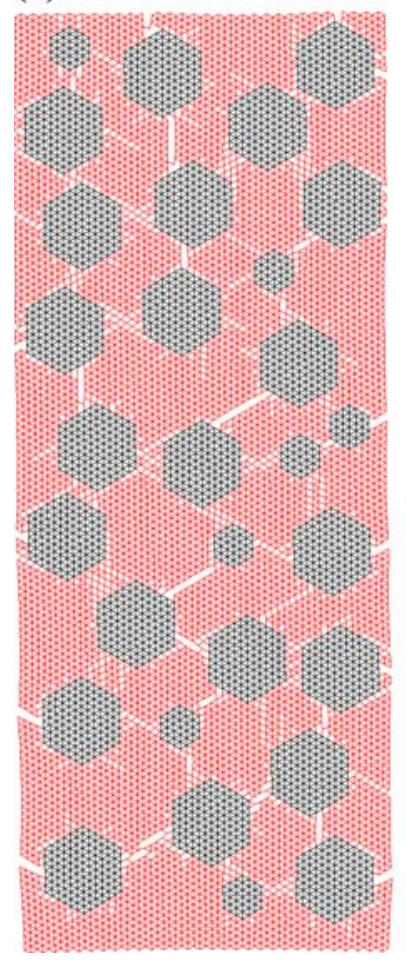

(c)

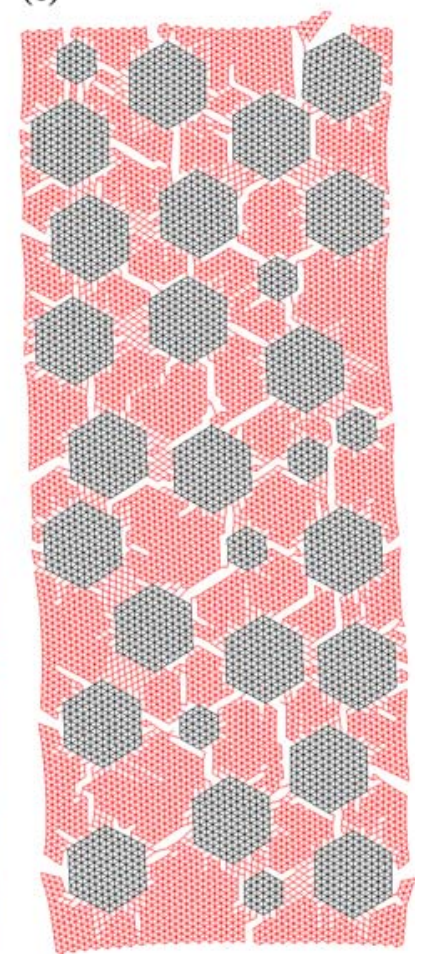

Fig. 13 In case 4, a the cumulative number of cracked elements versus displacement; and crack patterns at two levels: b level $a$; $\mathbf{c}$ level $b$

mentioned in Case 1. Therefore, average distance between adjacent aggregates in concrete can be taken as a characteristic length of shrinkage-induced cracks inside specimen. When comparing Fig. 13b, c with Fig. 8b-d, it can be found that the crack pattern in Case 4 is more smashed than Case 1. The reason is that the higher aggregate content in Case 4 decreases the average distance of adjacent aggregates. In Case 3, decrease of aggregate size increases this average distance, leading to longer shrinkage-induced cracks (Fig. 12d).

From Fig. 13a, the total of failures in Case 4 increases more quickly with shrinkage rate than in Case 1 . This can be explained as follows. As mentioned in Case 3, the total length

of "mismatch lines" corresponds to the sum of aggregates' perimeters, therefore, a higher aggregate content increases this total length. Because the mismatch line is the dominant source of failures, the bigger their total length becomes, the easier and more frequently shrinkage-induced cracking happens.

\section{Conclusions}

A numerical method to simulate shrinkage-induced cracking in cement-based composites was proposed. Regardless of moisture transport, temperature and so on, the problem is simplified to a pure mechanical process. No obvious external load serves as the drive to propel the shrinkage-induced deformation, therefore, it is hard to simulate by classical finite element method. As a solution, the mismatch displacement field is calculated by constructing two virtual displacement fields under the assumption of uniform matrix shrinkage. Then, numerical examples were given. Influences of different factors on failure processes were analyzed.

From the numerical results, shrinkage-induced cracks distribute all over the specimen as well as aggregates. Many of these cracks are as long as or even longer than the characteristic aggregate size under the current parameter setting. Furthermore, shrinkage leads to a non-zero stress field in specimen. Shrinkage-induced cracks and the pre-existing stress field can also play an important role in macro-level properties as well as particle structure, which will be a future topic.

Acknowledgments This research work was sponsored by National Natural Science Foundation of China through Grant Nos. 10572140, 10232050 and 10721202.

\section{References}

1. Bazant ZP, Tabbara MR, Kazemi MT, Cabot GR (1990) Random particle model for fracture of aggregate or fiber composites. J Eng Mech 116(8):1686-1705

2. Bolander JE Jr, Saito S (1998) Fracture analysis using spring network with random geometry. Eng Fract Mech 61(5-6):569-591

3. Bolander JE Jr, Berton S (2004) Simulation of shrinkage induced cracking in cement composite overlays. Cem Concr Compos 26:861-871

4. Cusatis G, Bazant ZPF, Cedolin LM (2003) Confinement-shear lattice model for conrete damage in tension and compression: I. Theory. J Eng Mech 129(12):1439-1448

5. Deshpande VS, Fleck NA, Ashby MF (2001) Effective properties of the octet-truss lattice material. J Mech Phys Solids 49:17471769

6. Fung YC (1977) A first course in continuum mechanics. PrenticeHall, New Jersey

7. Gao HJ, Klein P (1998) Numerical simulation of crack growth in an isotropic solid with randomized internal cohesive bonds. J Mech Phys Solids 46(2):187-218 
8. Gilbert RI (2005) AS3600 creep and shrinkage models for normal and high strength concrete. In: Gardner NJ, Weiss J (eds) Shrinkage and creep of concrete. Farmington Hills, Michigan

9. Herrmann HJ, Roux S (1992) Statistical models for the fracture of disordered media. Elsevier, Amsterdam

10. Hrennikoff A (1941) Solution of problems of elasticity by the framework method. J Appl Mech 12:169-175

11. Hughes TJR, Taylor RL, Kanoknukulchai S (1977) A simple and efficient finite element for bending. Int J Numer Eng 11: 1529-1543

12. John DA St, Poole AW, Sims I (1998) Concrete petrography: a handbook of investigative techniques. Wiley, New York

13. Karihaloo BL, Shao PF, Xiao QZ (2003) Lattice modelling of the failure of particle composites. Eng Fract Mech 70:2385-2406

14. Krajcinovic D (2000) Damage mechanics: accomplishments, trends and needs. Int J Solids Struct 37:267-277

15. Lura PJ, Mejlede jensen O, Ye G, Tanaka K (2005) Identification of microcracks caused by autogenous shrinkage. In: s.n. (ed) Proceedings of the international workshop on materials science in $21 \mathrm{st}$ century for the construction industry-durability, repair and recycling of concrete structures. Sapporo, Japan

16. Lemaitre J (1996) A course on damage mechanics. Science Press, Beijing (in Chinese)

17. Lilliu G, van Mier JGM (2003) 3D lattice type fracture model for concrete. Eng Fract Mech 70:927-941

18. Liu JX, Deng SC, Zhang J, Liang NG (2007) Lattice type of fracture model for concrete. Theor Appl Fract Mech 48:269-284

19. Liu JX, Deng SC, Liang NG (2008) Comparison of the quasi-static method and the dynamic method for simulating fracture processes in concrete. Comput Mech 41:647-660

20. Liu JX, Zhao ZY, Deng SC, Liang NG (2008) Numerical investigation of crack growth in concrete subjected to compression by the generalized beam lattice model. Comput Mech. doi:10.1007/ s00466-008-0305-z
21. Mckown S, Shen Y, Brookes WK et al (2008) The quasi-static and blast loading response of lattice structures. Int J Impact Eng 35:795-810

22. Ostoja-Starzewski M (2002) Lattice models in micromechanics. Appl Mech Rev 55:35-60

23. Ostoja-Starzewski M (2007) Microstructural randomness and scaling in mechanics of materials. Chapman \& Hall/CRC, London/Boca Raton

24. Potyondy DO, Cundall PA (2004) A bonded-particle model for rock. Int J Rock Mech Min Sci 41:1329-1364

25. Rots JG, Belletti B, Invernizzi S (2008) Robust modeling of RC structures with an "event-by-event" strategy. Eng Fract Mech 75(3-4):590-614

26. Salganik RL, Gotlib VA (2000) Continuum versus discontinuum damage mechanics of creep caused by microcracking. Int J Fract 101:181-201

27. Schlangen E, van Mier JGM (1992) Experimental and numerical analysis of the micromechanics of fracture of cement-based composites. Cem Concr Compos 14(2):105-118

28. Schorn H, Rode U (1987) 3-D modeling of process zone in concrete by numerical simulation. In: Shah SP, Swartz SE (eds) Fracture of concrete and rock. Springer, New York pp 220-228

29. van Mier JGM (1997) Fracture processes of concrete. CRC Press, Boca Raton

30. van Mier JGM, van Vliet MRA, Wang TK (2002) Fracture mechanisms in particle composites: statistical aspects in lattice type analysis. Mech Mater 34:705-724

31. Wang XC (2003) Finite element method, 1st edn. Tsinghua University Press, Beijing 